\title{
Functional Attributes Discriminating Mechano-Insensitive and Mechano-Responsive C Nociceptors in Human Skin
}

\author{
C. Weidner, ${ }^{1}$ M. Schmelz, ${ }^{1}$ R. Schmidt, ${ }^{2}$ B. Hansson, ${ }^{2}$ H. O. Handwerker, ${ }^{1}$ H. E. Torebjörk ${ }^{2}$ \\ ${ }^{1}$ Department of Physiology and Experimental Pathophysiology, University of Erlangen/Nürnberg, D-91054 Erlangen, \\ Germany, and 2Department of Clinical Neurophysiology, University of Uppsala, S-75185 Uppsala, Sweden
}

Microneurography was used in healthy human subjects to record action potentials from unmyelinated nerve fibers $(C$ units) in cutaneous fascicles of the peroneal nerve.

Activity-dependent slowing $(n=96)$ and transcutaneous electrical thresholds ( $n=67$ ) were determined. Eight units were sympathetic efferents according to their responses to sympathetic reflex provocations. Mechano-heat-responsive $\mathrm{C}$ units $(\mathrm{CMH})(n=56)$ had thresholds to von Frey hair stimulation $\leq 90$ $\mathrm{mN}$ (6.5 bar). Mechano-insensitive $\mathrm{C}$ units $(n=32)$ were unresponsive to $750 \mathrm{mN}$ (18 bar). Twenty-six mechano-insensitive units responded to heat $(\mathrm{CH})$, and the remaining six units did not respond to physical stimuli but were proven to be afferent by their response to intracutaneous capsaicin $\left(\mathrm{CM}_{\mathrm{i}} \mathrm{H}_{\mathrm{i}}\right)$.

Mechano-insensitive units had significantly slower conduction velocity $(0.81 \pm 0.03 \mathrm{~m} / \mathrm{sec})$, and $\mathrm{CH}$ units had higher heat thresholds $\left(48.0 \pm 0.6^{\circ} \mathrm{C}\right)$ compared with $\mathrm{CMH}$ units $(1.01 \pm$ $0.01 \mathrm{~m} / \mathrm{sec} ; 40.7 \pm 0.4^{\circ} \mathrm{C}$ ). Transcutaneous electrical thresholds were $<9 \mathrm{~mA}$ for $\mathrm{CMH}$ units and $>35 \mathrm{~mA}$ for $\mathrm{CH}$ and $\mathrm{CM}_{\mathrm{i}} \mathrm{H}_{\mathrm{i}}$ units. Activity-dependent slowing was much more pronounced in mechano-insensitive than in mechano-responsive units, without overlap. Sympathetic efferent $\mathrm{C}$ units showed intermediate slowing, significantly different from $\mathrm{CMH}$, and completely separate from $\mathrm{CH}$ and $\mathrm{CM}_{\mathrm{i}} \mathrm{H}_{\mathrm{i}}$ units. The activity-dependent slowing of conduction provides evidence for different membrane attributes of different classes of $\mathrm{C}$ fibers in humans.

Key words: mechanosensitivity; human C fibers; activationdependent slowing; microneurography; afterhyperpolarization; pain
$\mathrm{C}$ nociceptors in human skin often have been regarded as a homogenous group of afferents responding to mechanical, thermal, and chemical stimuli. In parallel to nociceptors found in animals, they have been named "polymodal" nociceptors to account for their responsiveness to different stimulus modalities (Bessou and Perl, 1969). This concept has been shown to be simplistic. Different classes of $\mathrm{C}$ nociceptors have been described in animals subserving separate functions. In particular, the different roles of mechano-responsive and mechano-insensitive nociceptors have been pointed out (Meyer and Campbell, 1981; Garell et al., 1996). By using new methods in microneurography, recently a considerable proportion of human $\mathrm{C}$ nociceptors has been found that differs from polymodal nociceptors by being insensitive to mechanical stimulation (Schmidt et al., 1995; Schmelz et al., 1997a). Some of these mechano-insensitive units do not respond to heating either and thus are regarded as "silent" nociceptors or $\mathrm{CM}_{\mathrm{i}} \mathrm{H}_{\mathrm{i}}$ (mechano- and heat-insensitive $\mathrm{C}$ unit), whereas others respond to heating and are named $\mathrm{CH}$ (heatresponsive $\mathrm{C}$ units). Many of these mechano-insensitive $\mathrm{C}$ units respond to capsaicin (Schmelz et al., 1999) and often become responsive to natural stimuli, such as tonic pressure and heat, when sensitized (Schmidt et al., 1995). For this reason, they have

Received June 1, 1999; revised Sept. 8, 1999; accepted Sept. 9, 1999.

This work was supported by a Max-Planck Price grant to H.E.T., Deutsche Forschungsgemeinschaft Grant Sonderforschungsbereich 353, Swedish Medical Research Council Project 5206, and a grant to R.S. from the Swedish Foundation for Brain Research.

Correspondence should be addressed to Prof. H. O. Handwerker, Institut für Physiologie und Experimentelle Pathophysiologie, Universität Erlangen/Nürnberg, Universitätsstradße 17, D-91054 Erlangen, Germany. E-mail: handwerker@ physiologie1.uni-erlangen.de.

Copyright (C) 1999 Society for Neuroscience 0270-6474/99/1910184-07\$05.00/0 been named "sleeping” nociceptors (Schaible and Schmidt, 1988). From these studies, it appears that, in human skin, as in other species and tissues, separate classes of nociceptors subserve different functions in pain and hyperalgesia.

Here, we studied whether functionally different classes of $\mathrm{C}$ nociceptors (i.e., $\mathrm{CMH}, \mathrm{CH}$, and $\mathrm{CM}_{\mathrm{i}} \mathrm{H}_{\mathrm{i}}$ ) can be separated by additional attributes unrelated to their mechanical or heat responsiveness.

Parts of the results have been published previously in abstract form (Weidner et al., 1998).

\section{MATERIALS AND METHODS}

Ninety-six C fiber units were recorded from cutaneous fascicles of the peroneal nerve by means of microneurography in our research laboratories in Uppsala, Sweden and Erlangen, Germany. Sixty-one subjects (42 male, 19 female), aged 19-32 years, participated in the study after giving their informed written consent. None of the subjects suffered from a relevant dermatological or neurological disease at the time of the study. The study was approved by the local ethics committees.

The microneurographic technique for recording from human $\mathrm{C}$ fibers has been described in detail previously (Torebjörk, 1974) and will only be summarized briefly. A microelectrode ( $0.2 \mathrm{~mm}$ in diameter) was manually inserted into the peroneal nerve dorsolateral to the fibular head for recording nerve signals, and a reference microelectrode was placed subcutaneously nearby. The uninsulated tip of the recording electrode was inserted in a cutaneous fascicle while its output signal was passed through an audio-amplifier. Positioning of the electrode was guided by the characteristic sound of multifiber discharges evoked by gently stroking the skin in the expected innervation territory (lower leg or foot dorsum).

Single electrical impulses $(0.2 \mathrm{msec}, 30-50 \mathrm{~mA}$ from an insulated constant current stimulator) (Digitimer DS7; Digitimer, Hertfordshire, UK) were then applied from a pointed steel probe with a small contact surface (1 $\mathrm{mm}$ in diameter), which was moved on the skin until single $\mathrm{C}$ unit responses, characterized by their long latencies, were obtained. 
When the skin innervation territory of a $\mathrm{C}$ fiber was found, two needle electrodes $(0.2 \mathrm{~mm}$ shaft diameter $)$ were inserted $5 \mathrm{~mm}$ apart in this territory for repetitive intracutaneous electrical stimulation $[0.25 \mathrm{~Hz}, 0.2$ msec, $10-150 \mathrm{~V}$, from an insulated Grass Instruments (Quincy, MA) S 88 stimulator]. C units were then characterized by the "marking" technique (Torebjörk and Hallin, 1974; Schmidt et al., 1995), i.e., any suddenly increased delay of the responses to these electrical stimuli was regarded as sign of activation of the respective unit. Sympathetic $C$ units were identified by their marking response during activation related to arousal stimuli, e.g., unexpected loud noise or mental stress, or during deep inspiration, stimuli, and maneuvers, which are known to elicit sympathetic reflexes in human skin nerves (Torebjörk and Hallin, 1970; Hagbarth et al., 1972; Hallin and Torebjörk, 1974). Afferent C units were identified by their marking response to natural stimulation or capsaicin injection inside their innervation territories in the skin.

A set of calibrated von Frey nylon monofilaments (Stoelting Co., Chicago, IL) was used to quantify mechanical thresholds. The forces exerted by the monofilaments were $1.5-750 \mathrm{mN}(0.8-18$ bar; tip diameter of $0.15-0.71 \mathrm{~mm})$, spaced by a factor of 2 or less. The lowest force that could induce marking responses from the receptive field of a unit was regarded as the mechanical threshold.

Heat stimuli were delivered from a halogen lamp feedback controlled by a thermocouple attached to the skin (Beck et al., 1974). Skin temperature was increased by $0.25^{\circ} \mathrm{C} / \mathrm{sec}$ from an adapting level of $32^{\circ} \mathrm{C}$ to tolerance level of $52^{\circ} \mathrm{C}$. Activation threshold of a $\mathrm{C}$ unit was determined from the first occurrence of marking.

Conduction velocity measurements. The latencies of $\mathrm{C}$ fiber responses to the first electrical impulse delivered from the intracutaneous needle electrodes after a rest period of at least 2 min were used for computing the conduction velocities (c.v.s.). The shortest distance between the stimulating needles in the skin and the recording electrode in the nerve was assessed with a measuring tape of millimeter accuracy. Room temperature was kept constant at $22-24^{\circ} \mathrm{C}$ throughout the experiments.

Transcutaneous electrical thresholds. From previous experience, we got the impression that thresholds for transcutaneous electrical stimulation might differentiate between different classes of $\mathrm{C}$ units, in contrast to intracutaneous stimulation depending more on the position of the needles relative to the respective nerve terminals.

To standardize the transcutaneous electrical thresholds, we used an applicator that was originally developed for localized iontophoresis. This probe provided a homogeneous electrical field little influenced by the contact pressure or the humidity of the skin. It consists of a Perspex holder containing in its cavity a round cotton disk, $5 \mathrm{~mm}$ in diameter, which is soaked with saline. This cotton disk was gently pressed to the skin by hand (Magerl et al., 1990). A Digitimer DS7 constant current stimulator was used to deliver single pulses of $0.2 \mathrm{msec}$. A large $(5 \times 10$ $\mathrm{cm})$ metal plate attached to the skin on the lower leg served as reference electrode. The electrical thresholds obtained with this probe did not change when the contact pressure was moderately varied or when the probe was slightly shifted within the innervation territory of the respective unit.

Activity-dependent slowing is a well known attribute of unmyelinated nerve fibers and also the basis of the marking technique. After a rest period of at least $2 \mathrm{~min}$, intracutaneous electrical stimuli were applied at increasing frequencies. The first stimulus train consisted of 20 pulses at $0.125 \mathrm{~Hz}$, immediately followed by a second train of 20 pulses at $0.25 \mathrm{~Hz}$, and a third of 30 pulses at $0.5 \mathrm{~Hz}$. The difference of conduction delays before and after these trains was evaluated for each frequency ("slowing"; see Figs. 2, 3) and also for the accumulated slowing during the entire stimulation protocol ("total slowing"; see Figs. 4, 5). In sympathetic units, the slowing was only evaluated when spontaneous activity was below one per minute.

Data acquisition and analysis. Signals from the recording electrodes were amplified and recorded on line by a personal computer through an interface card (DAP; Microstar) and the SPIKE/SPIDI software package (Forster and Handwerker, 1990). Standard ANOVA, ANCOVA, and linear regression tests were performed (StatSoft Inc., Tulsa, OK). When appropriate, nonparametric statistics were applied. Differences were regarded to be significant at $p<0.05$ and $p<0.01$, respectively. The appropriate corrections (Bonferroni) for repetitive testing were used.

\section{RESULTS}

\section{Classification of $\mathbf{C}$ units}

Ninety-six C units were recorded with innervation territories on the lower leg and foot. Conduction distances were $220-510 \mathrm{~mm}$ (mean of $362 \mathrm{~mm}$ ). Fifty-six units that responded to stimulation with von Frey hairs and to heating up to $52^{\circ} \mathrm{C}$ were classified as $\mathrm{CMH}$ (polymodal nociceptors). Twenty-six units that were found responsive to heating but not to mechanical forces up to $750 \mathrm{mN}$ (18 bar) were classified as $\mathrm{CH}$ units, and six units that were responsive to neither strong mechanical nor heat stimuli in noninflamed skin were classified as $\mathrm{CM}_{\mathrm{i}} \mathrm{H}_{\mathrm{i}}$. They were proven to be afferent by their responsiveness to intracutaneous injection of capsaicin $(0.1 \% ; 20 \mu \mathrm{l})$ within their innervation territory. Eight units were classified as sympathetic units responding to arousal stimuli. The proportion of mechano-responsive and mechanoinsensitive units was similar in male (36/20) and female (20/12) subjects ( $\chi^{2}$ test; NS).

Mechanical thresholds of $\mathrm{CMH}$ units (polymodal $\mathrm{C}$ fibers) were in the range of 7-90 $\mathrm{mN}$ (median of $30 \mathrm{mN}$; upper and lower quartiles, 22 and $43 \mathrm{mN}$, respectively). This range of thresholds of $\mathrm{CMH}$ units to von Frey hair stimulation is comparable with that published in previous studies (Adriaensen et al., 1983). A threshold of $750 \mathrm{mN}$ (18 bar) was arbitrarily set as the cutoff value between mechano-responsive and mechano-insensitive units. The latter were not even excited by insertion of the stimulation needles or a hypodermic needle in the innervation territory.

Heat thresholds of mechano-responsive $\mathrm{CMH}$ units were $40.7 \pm 3.0^{\circ} \mathrm{C}($ mean $\pm \mathrm{SEM})(n=51)$. Mean heat thresholds of mechano-insensitive $\mathrm{CH}$ units were significantly higher at $48.0 \pm$ $3.0^{\circ} \mathrm{C}(n=25 ; p<0.01$; unpaired $t$ test) (see Fig. 6).

\section{Conduction velocities}

The initial c.v.s of the $\mathrm{CMH}$ units ranged from 0.79 to $1.27 \mathrm{~m} / \mathrm{sec}$ (mean of $1.01 \mathrm{~m} / \mathrm{sec}$ ). The c.v.s of $\mathrm{CH}$-units ranged from 0.59 to $1.19 \mathrm{~m} / \mathrm{sec}$ (mean of $0.87 \mathrm{~m} / \mathrm{sec}$ ), and $\mathrm{CM}_{\mathrm{i}} \mathrm{H}_{\mathrm{i}}$ units had conduction velocities ranging from 0.49 to $1.24 \mathrm{~m} / \mathrm{sec}$ (mean of $0.82 \mathrm{~m} / \mathrm{sec}$ ). The c.v.s of mechano-insensitive $\mathrm{CM}_{\mathrm{i}} \mathrm{H}_{\mathrm{i}}$ and $\mathrm{CH}$ units were significantly lower than those of the mechano-responsive units ( $p<0.01 ; t$ test) as shown in previous studies (Schmidt et al., 1995; Schmelz et al., 1997a). However, there was no significant difference of c.v.s between $\mathrm{CH}$ and $\mathrm{CM}_{\mathrm{i}} \mathrm{H}_{\mathrm{i}}(p>0.05 ; t$ test $)$. Sympathetic efferent units had c.v.s ranging from 0.73 to 0.85 $\mathrm{m} / \mathrm{sec}$ (mean of $0.78 \mathrm{~m} / \mathrm{sec}$ ).

\section{Thresholds to transcutaneous electrical stimulation}

The stimulus probe described in Materials and Methods provided a uniform current field through a saline bridge and was used for assessment of the transcutaneous electrical thresholds in 67 afferent $\mathrm{C}$ units. Forty-six CMH units had a median threshold of $4.0 \mathrm{~mA}$ (2.0 and 5.5, lower and upper quartile, respectively). For the 21 mechano-insensitive units, the threshold was $60 \mathrm{~mA}$ on average. In seven mechano-insensitive units, the threshold exceeded $80 \mathrm{~mA}$ and could not be determined, because the stimulus intensity exceeded the tolerance limit of the subject. The difference between mechano-responsive and mechano-insensitive units was striking ( $p<0.001$; Mann-Whitney $U$ test) (Fig. 1).

Conduction velocity was negatively correlated with transcutaneous electrical thresholds ( $r=0.41 ; p<0.01$; linear regression). There was no significant correlation, however, between these two parameters within the subgroups of mechano-responsive $(r=$ $0.01 ; p=0.9)$ or mechano-insensitive $(r=0.02 ; p=0.6)$ units. Therefore, the negative correlation just reflects that both parameters, conduction velocity and transcutaneous electrical threshold, differ between the two classes of mechano-responsive and mechano-insensitive units. 


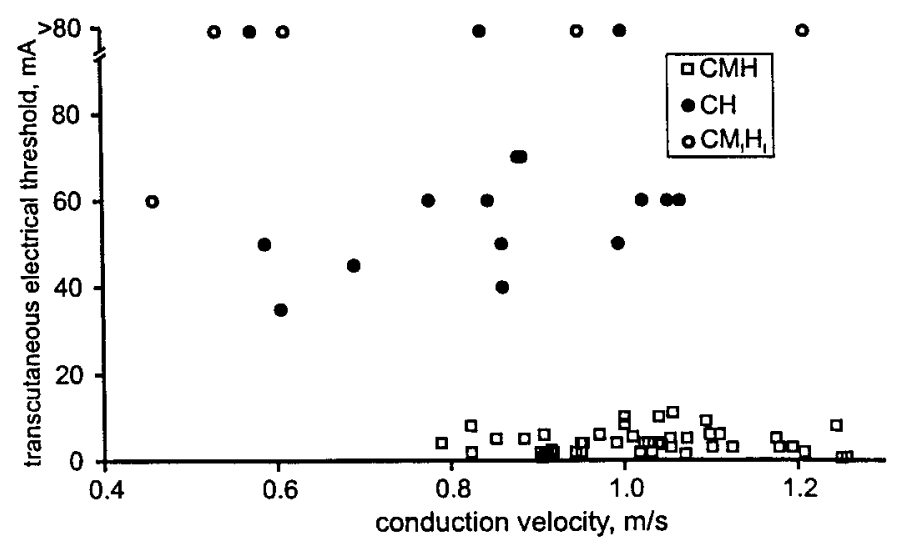

Figure 1. Conduction velocity and electrical thresholds. Transcutaneous electrical thresholds were assessed with a surface electrode designed for homogeneous current application. Forty-six CMHs (open squares), 16 $\mathrm{CHs}$ ( filled circles), and five $\mathrm{CM}_{\mathrm{i}} \mathrm{H}_{\mathrm{i}} \mathrm{s}$ (open circles) were tested. The difference between transcutaneous electrical thresholds of mechanoresponsive (squares) and mechano-insensitive (circles) units was highly significant. Within each category of units, no significant correlations between conduction velocities and transcutaneous electrical thresholds were found.

\section{Activity-dependent slowing}

Repetitive electrical stimulation at $0.125,0.25$, and $0.5 \mathrm{~Hz}$ gradually increased the response latencies in C fibers. As shown in the specimen in Figure 2, this increase differed between nociceptor classes. Response latency of the mechano-insensitive fiber $(b)$ markedly increased during stimulation at low frequencies, whereas that of the mechano-responsive fibers remained fairly stable.

The slowing of mechano-insensitive units by far exceeded that of mechano-responsive ones at each stimulus frequency $(p<$ $0.0001 ; t$ test) (Fig. 3). The two lower stimulation frequencies $(0.125$ and $0.25 \mathrm{~Hz})$ produced only moderate slowing in mechanoresponsive units, i.e., $20.7 \pm 6.1 \%$ of the total slowing (the increase in response latency during the entire electrical protocol). In contrast, among the mechano-insensitive units, approximately half of the total slowing was induced at the lower stimulation frequencies $\left(50.5 \pm 2.8 \%\right.$ in $\mathrm{CM}_{\mathrm{i}} \mathrm{H}_{\mathrm{i}} \mathrm{s} ; 49.7 \pm 1.2 \%$ in $\left.\mathrm{CHs}\right)$. The differences between the two classes of mechano-insensitive units were not significant, and hence mechano-insensitive units were regarded as one group in the following analysis. The slowing of sympathetic fibers was intermediate and significantly different from the mechano-responsive and mechano-insensitive fibers at all stimulation frequencies $(p<0.0001)$. For the two lowest frequencies, the slowing of the sympathetic fibers amounted to $35.3 \pm 3.1 \%$ of the total slowing.

\section{Slowing and receptive thresholds}

In Figure 4, $A$ and $C$, it appears as if there were positive correlations between total slowing and heat and electrical thresholds, respectively. However, this is entirely attributable to the higher heat and electrical thresholds of mechano-insensitive units. There was no significant correlation between the total slowing and heat thresholds within mechano-responsive $(r=0.2 ; p=0.15$; linear regression) or mechano-insensitive $(r=-0.1 ; p=0.6)$ units. Likewise, within the groups of nociceptors, no significant correlation between total slowing and electrical thresholds was obtained in mechano-responsive $(r=0.01$; NS) and mechanoinsensitive $(r=-0.05$; NS) fibers.

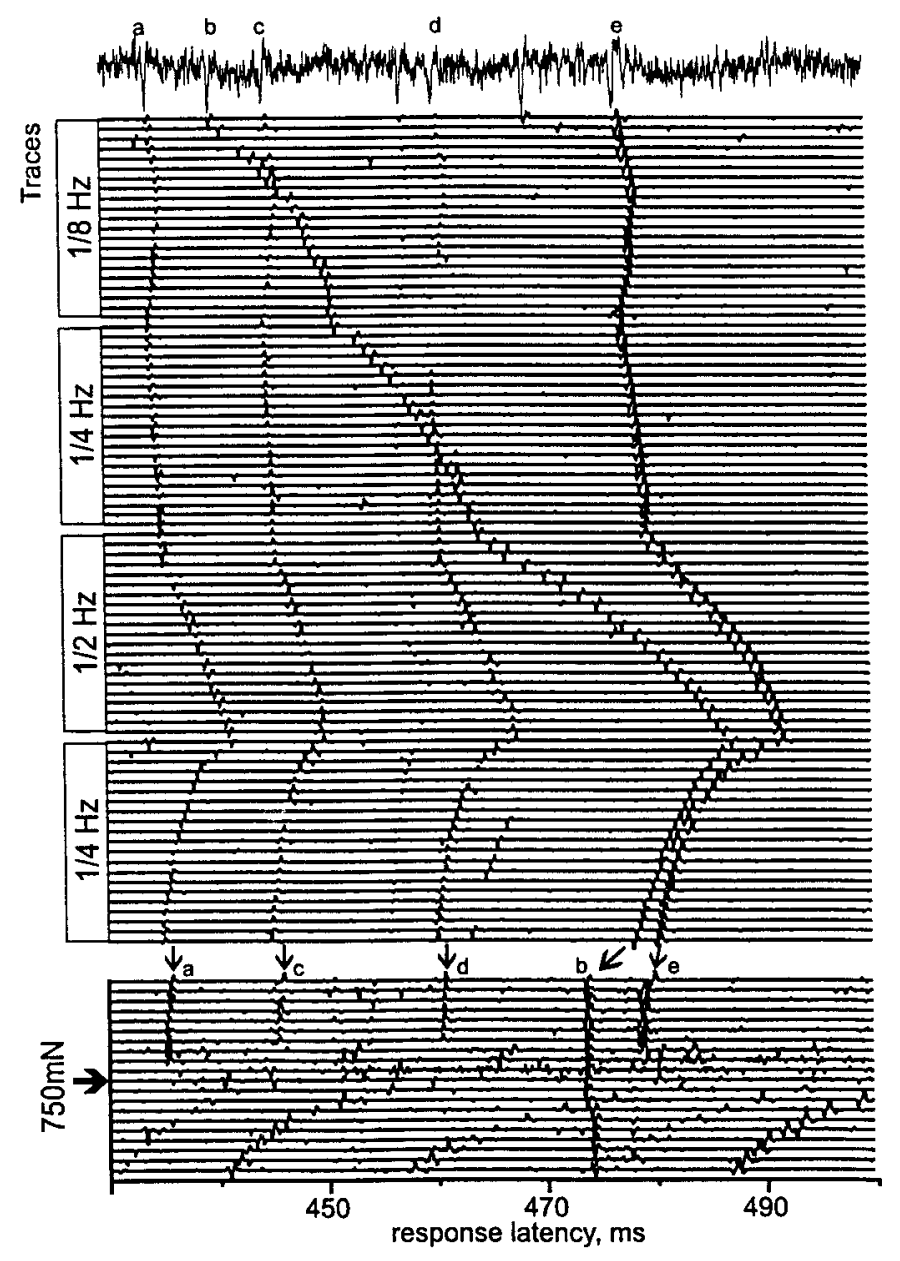

Figure 2. Slowing of conduction velocity during repetitive stimulation. Specimen obtained during the electrical stimulation protocol applied to a group of five $\mathrm{C}$ units recorded simultaneously. After a 2 min recovery period, the nerve terminals were stimulated by intracutaneous impulses at $0.125,0.25$, and $0.5 \mathrm{~Hz}$ and then again at $0.25 \mathrm{~Hz}$. Subsequent traces are shown from top to bottom. The top trace provides an amplified and unfiltered view of the recording also shown in the first trace of the following sequence. For the standard protocol, 20 stimuli were applied at 0.125 and $0.25 \mathrm{~Hz}$, and 30 stimuli at $0.5 \mathrm{~Hz}$. In this specimen record, only 20 stimuli at $0.5 \mathrm{~Hz}$ are shown. In the bottom, responses of the five units to mechanical stimulation with a stiff von Frey bristle are shown $(750 \mathrm{mN}$; arrow). Unit $(b)$ was mechano-insensitive. Units $(a, c-e)$ showed pronounced marking responses to mechanical stimulation.

Mechanical thresholds could be assessed only in the group of mechano-responsive units. In this group, a trend toward more pronounced slowing was found for units with higher mechanical thresholds ( $r=0.33 ; p=0.05$; linear regression).

\section{Conduction velocity and slowing}

Generally, conduction velocity was inversely correlated with the increase in response latency (total slowing) during repetitive electrical stimulation ( $r=-0.65 ; p<0.001$; linear regression). However, the effect of conduction velocity did not fully account for the difference in slowing between the mechano-responsive and mechano-insensitive units. When the effect of conduction velocity was controlled by introducing it as a covariate in a MANCOVA, the slowing was still significantly different between mechano-insensitive and mechano-responsive units $(p<0.0001$; MANCOVA). Thus, both parameters, slowing and conduction 


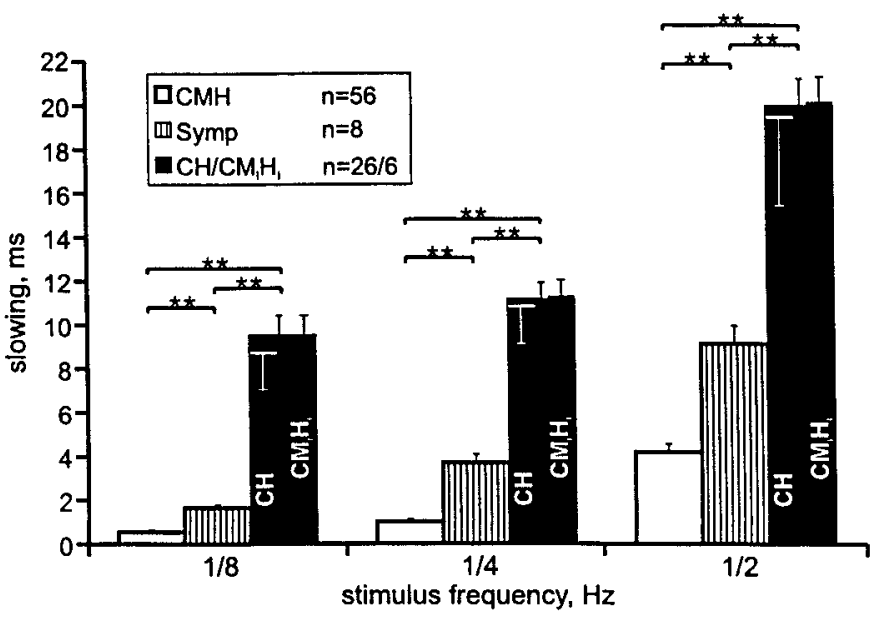

Figure 3. Slowing in three categories of C-fibers. Latency increases (mean \pm SEM) induced by 20 impulses at 0.125 and $0.25 \mathrm{~Hz}$ and after 30 pulses at $0.5 \mathrm{~Hz}$ are shown separately for each frequency. Within the group of mechano-insensitive units ( filled bars), values for $\mathrm{CH}$ and $\mathrm{CM}_{\mathrm{i}} \mathrm{H}_{\mathrm{i}}$ are shown separately. There was no statistically significant difference between the two subgroups of mechano-insensitive units. Slowing differed significantly between the $\mathrm{CMH}$, sympathetic, and $\mathrm{CH} / \mathrm{CMiHi}$ unit categories. Statistical differences were assessed by unpaired $t$ tests; ${ }^{*} p<0.05$; $* * p<0.01$

velocity, represented independent criteria for discriminating between these two categories of afferent $\mathrm{C}$ fibers. When the increase of response latency (total slowing) was plotted against conduction velocity, mechano-responsive and mechano-insensitive units formed separate clusters without any overlap (Fig. 5). Similarly, the sympathetic units were clustered without overlapping the mechano-insensitive fiber group. In contrast, no clear distinction between sympathetic and mechano-receptive units was apparent.

\section{DISCUSSION}

\section{Separation of the $\mathbf{C}$ nociceptors in human skin in two categories}

The results of this study demonstrate that afferent $\mathrm{C}$ fibers in human skin can be separated in two groups on the basis of their mechanical responsiveness. Using graded von Frey bristles for semiquantitative assessment of mechanical thresholds, this separation was complete (in noninflamed skin) because the mechanoinsensitive units could not be excited with forces nine times the strength of the strongest forces (three times the highest pressure) required for exciting mechano-heat responsive $(\mathrm{CMH}$, polymodal) nociceptors. Moreover, the mechano-insensitive units did not even respond to needle insertion. Thus, they were completely mechano-insensitive, and the cutoff force of $750 \mathrm{mN}$ (18 bar) was just an arbitrary limit set for routine testing. This fiber type in humans may not be completely identical with mechanoinsensitive afferents described in monkey, cat, or goat, which include units responding to forces exceeding $100 \mathrm{mN}$ (6 bar) or $160 \mathrm{mN}$ (Cooper et al., 1991; Davis et al., 1993; Garell et al., 1996).

The purpose of this study was to find out whether this separation extended also to other traits that were not directly related to mechanical responsiveness. Although significant differences in heat thresholds and conduction velocities were found, only transcutaneous electrical threshold and activation-dependent slowing

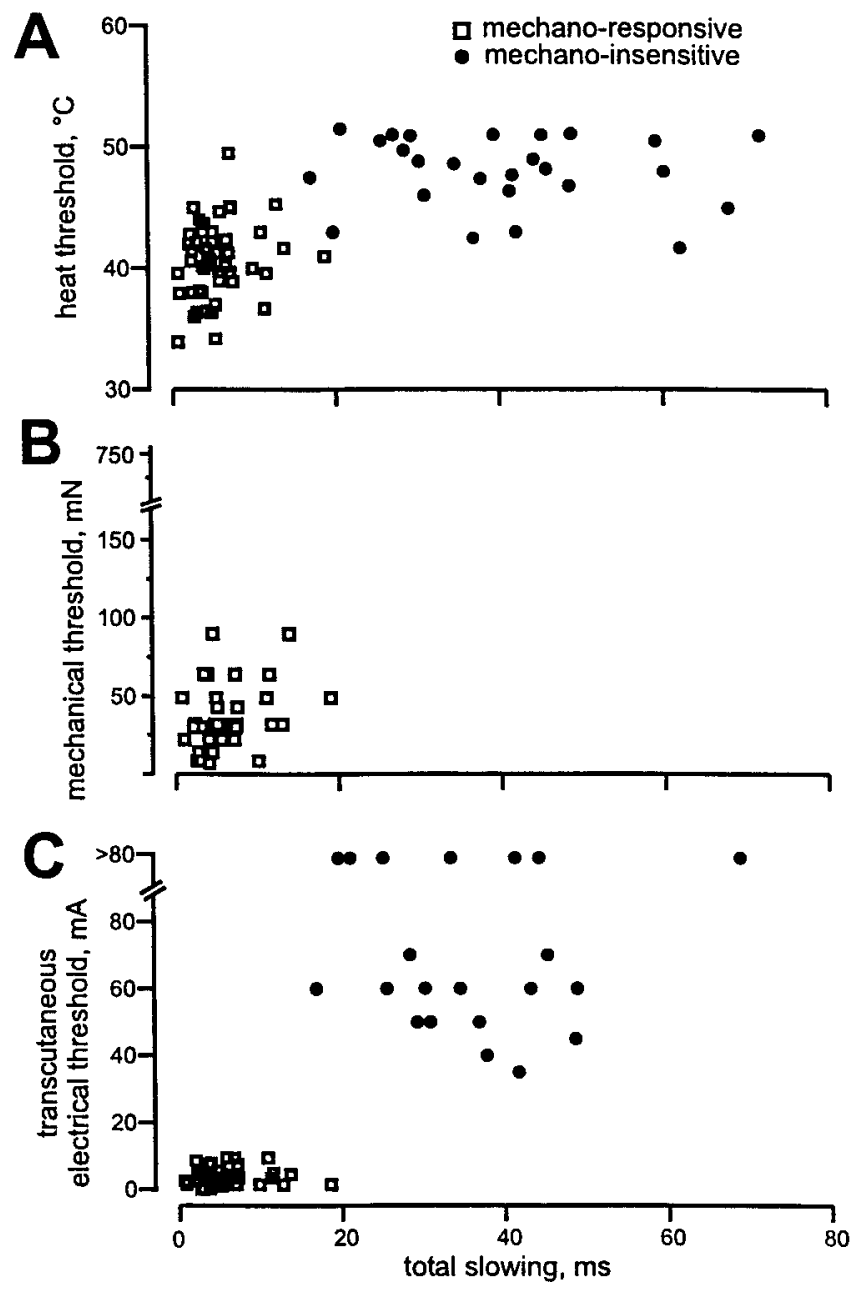

Figure 4. Thresholds of afferent units and total slowing. Heat $(A)$, mechanical $(B)$, and transcutaneous electrical thresholds $(C)$ are all plotted against total slowing (sum of latency increases during application of all three stimulus frequencies). Mechano-insensitive units (filled circles) did not respond to von Frey filament stimulation $(750 \mathrm{mN})$, and hence no mechanical thresholds are shown for this category. Heat thresholds were higher in mechano-insensitive units $(p<0.001$; unpaired $t$ test $)$ but did overlap between the two classes. In contrast, electrical thresholds separated both nociceptor classes completely. Within the categories of mechano-responsive (open squares) and mechano-insensitive units, none of the threshold values showed significant correlations with total slowing apart from a low positive correlation between total slowing and mechanical thresholds $(p=0.05 ; B)$.

of conduction completely separated mechano-responsive from mechano-insensitive units, as demonstrated in the present study.

Within the group of mechano-responsive units, there might be a trend toward more slowing in units with higher von Frey thresholds (Fig. 4B). However, this trend seemed to represent a continuum, and we could not in human hairy skin detect distinct subdivisions in both slowing and mechano-sensitivity in this group, as found with other parameters of responsiveness in cat (Garell et al., 1996) or goat (Cooper et al., 1991).

Conduction velocities were measured between the innervation territory in the skin and the recording site at knee level. From the initial conduction velocities, the three $\mathrm{C}$ unit categories were not clearly separated (Figs. 1, 5), although afferent mechanoinsensitive units tended to be slower than mechano-responsive units (Schmidt et al., 1995). Repetitive stimulation at a frequency 


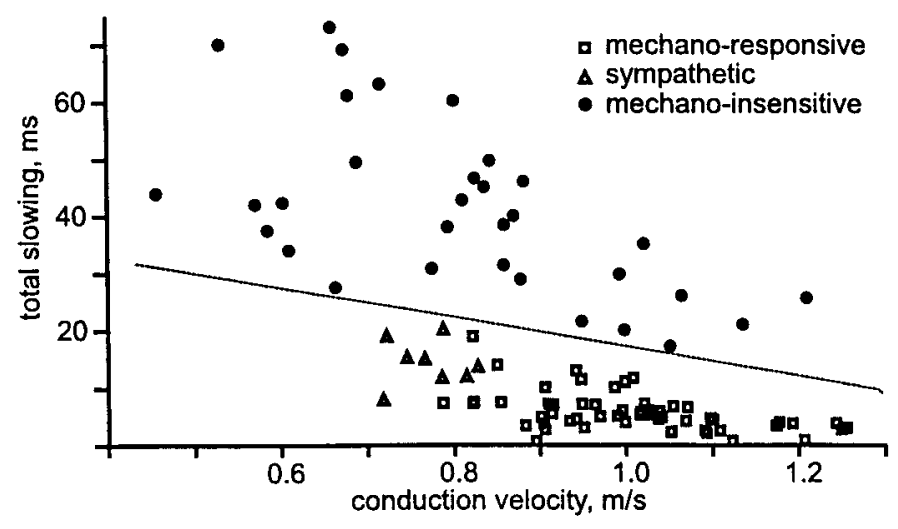

Figure 5. Total slowing from the three categories of $\mathrm{C}$ units plotted versus conduction velocities. The two afferent classes of mechanoresponsive and mechano-insensitive units formed two separate clusters (dotted line), but there was no clear separation between mechanoresponsive and sympathetic units.

of $0.25 \mathrm{~Hz}$ exaggerated this difference because activationdependent slowing was more pronounced in mechano-insensitive units. However, even under this condition, conduction velocity did not completely separate the two classes of nociceptors, suggesting that axonal diameter cannot be regarded as a major criterion for the differentiation.

A clear separation between the two categories of afferent $\mathrm{C}$ fibers was provided by the different thresholds to transcutaneous electrical stimulation (Fig. 1). The remarkably lower activation threshold of the mechano-responsive $\mathrm{CMH}$ units might be a result of a more superficial location or to a larger axon diameter, and hence membrane surface, at the site of action potential generation. However, as stated above, conduction velocity and hence axon diameter was not correlated to electrical thresholds within the groups of mechano-responsive and mechanoinsensitive units. Alternatively, density or activation threshold of sodium channels at the site of action potential generation might differ between the two groups. Regardless of possible mechanisms, the different electrical thresholds of the two nociceptor classes provides the exciting possibility to selectively stimulate only one subgroup of nociceptors and study their specific effects in awake humans.

Activation-dependent slowing of impulse conduction is more pronounced and prolonged in $\mathrm{C}$ fibers than in A fibers (Torebjörk and Hallin, 1974). In our present study, we showed that pronounced slowing occurred in mechano-insensitive $\mathrm{C}$ units at stimulus frequencies of only $0.125 \mathrm{~Hz}$. The phenomenon of activity-dependent slowing is probably related to long-term afterhyperpolarization (AHP) of the conductive membrane after axonal activation (Rang and Ritchie, 1968; Jansen and Nicholls, 1973). Evidence has been provided that elevated sodium extrusion by an electrogenic pump is partly responsible (Rang and Ritchie, 1968; Jansen and Nicholls, 1973; Van Essen, 1973). Ouabaine (an inhibitor of the $\mathrm{Na}^{+} / \mathrm{K}^{+}$pump) abolished the AHP in sensory neurons of the leech (Jansen and Nicholls, 1973; Van Essen, 1973) and activity-dependent threshold increase in frog sciatic nerve (Raymond and Lettvin, 1978), which again was closely related to conduction velocity slowing (Raymond and Lettvin, 1978). Recently, a calcium-gated $\mathrm{K}^{+}$channel was pro- posed as a further mechanism for AHP and activity-dependent slowing (Gee et al., 1996). On the other hand, AHP is known to gate a rectifying cationic inward current $\left(I_{\mathrm{h}}\right)$ in rat peripheral axons limiting it (Grafe et al., 1997). Blocking of $I_{\mathrm{h}}$ by ZD 7288 augmented the post-tetanic hyperpolarization and enhanced activity-dependent conduction velocity slowing in isolated rat dorsal spinal root (Grafe et al., 1997).

To explain the pronounced activity-dependent slowing in mechano-insensitive versus mechano-responsive fibers, one might assume either an increase in the AHP-promoting factors or a decrease in $I_{\mathrm{h}}$ capacity.

It has been reported before from rat saphenous $\mathrm{C}$ fibers that activity-dependent slowing could provide a tool for separating nociceptors from cold units (Raymond et al., 1990; Thalhammer et al., 1994). In another study in the rat, a bimodal distribution of conduction velocity slowing in silent (i.e., mechano-insensitive) units was observed (Gee et al., 1996). It was assumed that this distribution would reflect the separation between silent afferents and sympathetic efferent units, which were disconnected from their cell bodies with the recording technique used. After excluding supposed sympathetic units, the mechano-insensitive units in this rat study showed a slowing pattern that was not different from that of polymodal $\mathrm{C}$ nociceptors. This result is at variance with our findings but may be attributable to the high stimulation frequency of $20 \mathrm{~Hz}$ used by Gee and coworkers (1996). In our experiments, the largest difference between the two classes of afferent fibers was found at stimulation frequencies of $0.125 \mathrm{~Hz}$, when the slowing in mechano-insensitive units was 19 times that in CMHs. Increasing frequency to $0.5 \mathrm{~Hz}$ reduced this difference to a factor of 4 .

Interestingly, differences in activation-dependent slowing between mechano-responsive $\mathrm{C}$ nociceptors and non-nociceptive $\mathrm{C}$ cold units have been described recently in humans (Serra et al., 1999) using stimulation frequencies of 1,2 , and $4 \mathrm{~Hz}$. No overlap of slowing was observed between mechano-receptive nociceptors and non-nociceptive cold-sensitive $\mathrm{C}$ fibers. However, in that study, no mechano-insensitive fibers were encountered, and no differences in activation-dependent slowing among the $\mathrm{C}$ nociceptors were found.

In our sample, activity-dependent slowing separated completely between mechano-insensitive and mechano-responsive $\mathrm{C}$ fibers so that this criterion can be used for classifying units without applying "natural" stimuli to their terminals. Especially for nerve preparations in soft tissue, such as intestine, this might be of special interest because it avoids the application of potentially destructive forces for characterization.

The sympathetic fibers that were identified by their reflex responses to arousal stimuli probably consist of both vasoconstrictor and sudomotor units, as shown previously (Delius et al., 1972; Hagbarth et al., 1972). In contrast to afferent C nociceptors, the sympathetic fibers exhibit ongoing activity. Activity-dependent slowing in our protocol could only be measured in units that had a mean ongoing discharge frequency of less than one impulse per minute. This means that the selection of sympathetic fibers in our material was biased toward units with very low firing frequency. This justifies the comparison of their slowing with that of nociceptive $\mathrm{C}$ fibers without spontaneous activity. Furthermore, after priming all $\mathrm{C}$ fibers by electrical stimulation at $0.125 \mathrm{~Hz}$, the separation between mechano-insensitive and sympathetic units was still complete. The difference between mechano-responsive and sympathetic fibers was also significant but could not be used 
for unambiguous classification because of some overlap between the groups (Fig. 5).

\section{Hypothetical molecular basis of the group differences}

Recently, it has been shown that the terminal membrane of $\mathrm{C}$ fibers and of small DRG neurons in culture are equipped with tetrodotoxin resistant (TTX-R) subtypes of sodium channels (Dib-Hajj et al., 1998; Trezise et al., 1998), which probably play an important role in various forms of pain and hyperalgesia (Gold et al., 1996; Akopian et al., 1999). Some properties of TTX-R sodium channels show parallels with characteristics of mechanoinsensitive $\mathrm{C}$ nociceptors. Repetitive electrical stimulation induced a reduction of $\mathrm{Na}^{+}$currents at low frequencies of $0.4 \mathrm{~Hz}$ (Scholz et al., 1998), which could be a basis for conduction velocity slowing of mechano-insensitive units at low frequencies. One subtype of TTX-R sodium channels, SNS/PN3, is characterized by a rather high activation threshold and long inactivation time constant (Akopian et al., 1996; Sangameswaran et al., 1996). If the mechano-insensitive $\mathrm{C}$ units in human skin were equipped with TTX-R sodium channels with an elevated activation threshold, this would explain their high activation thresholds for transcutaneous electrical stimuli. Interestingly, blockers of TTX-R sodium channels have been shown to attenuate hyperalgesia without affecting acute pain thresholds in an animal study (Trezise et al., 1998), and similar results were obtained using low-dose lidocaine intravenously in human (Koppert et al., 1998). The antihyperalgesic effects observed in humans were restricted to models in which mechano-insensitive nociceptors are supposed to be of major relevance [i.e., tonic mechanical pressure (Schmelz et al., 1997b), histamine-induced itch (Schmelz et al., 1997a), and neurogenic flare (Lynn et al., 1996)].

In conclusion, two main subgroups of $\mathrm{C}$ nociceptors (mechanoresponsive and mechano-insensitive) exist in human skin that differ not only in their response characteristics but also in their electrical thresholds and activity-dependent slowing. The new finding that the mechano-insensitive $\mathrm{C}$ nociceptors have distinct membrane characteristics opens up possibilities for selective pharmacological interventions tailored for these $\mathrm{C}$ fibers, which may be of particular importance for the treatment of inflammatory pain and hyperalgesia.

\section{REFERENCES}

Adriaensen H, Gybels J, Handwerker HO, Van HJ (1983) Response properties of thin myelinated (A-delta) fibers in human skin nerves. J Neurophysiol 49:111-122.

Akopian AN, Sivilotti L, Wood JN (1996) A tetrodotoxin-resistant voltage-gated sodium channel expressed by sensory neurons. Nature 379:257-262.

Akopian AN, Souslova V, England S, Okuse K, Ogata J, Ure J, Smith A, Kerr BJ, Mcmahon SB, Boyce S, Hill R, Stafna LC, Dickenson AH, Wood JN (1999) The tetrodotoxin-resistant sodium channel SNS has a specialized function in pain pathways. Nat Neurosci 2:541-548.

Beck PW, Handwerker HO, Zimmermann M (1974) Nervous outflow from the cat's foot during noxious radiant heat stimulation. Brain Res 67:373-386.

Bessou P, Perl ER (1969) Responses of cutaneous sensory units with unmyelinated fibers to noxious stimuli. J Neurophysiol 32:1025-1043.

Cooper B, Ahlquist M, Friedman RM, Labanc J (1991) Properties of high-threshold mechanoreceptors in the goat oral mucosa. II. Dynamic and static reactivity in carrageenan-inflamed mucosa. J Neurophysiol 66:1280-1290.

Davis KD, Meyer RA, Campbell JN (1993) Chemosensitivity and sensitization of nociceptive afferents that innervate the hairy skin of monkey. J Neurophysiol 69:1071-1081.
Delius W, Hagbarth KE, Hongell A, Wallin BG (1972) Manoeuvres affecting sympathetic outflow in human skin nerves. Acta Physiol Scand 84:177-186.

Dib-Hajj SD, Tyrrell L, Black JA, Waxman SG (1998) NaN, a novel voltage-gated $\mathrm{Na}$ channel, is expressed preferentially in peripheral sensory neurons and down-regulated after axotomy. Proc Natl Acad Sci USA 95:8963-8968.

Forster C, Handwerker HO (1990) Automatic classification and analysis of microneurographic spike data using a PC/AT. J Neurosci Methods 31:109-118.

Garell PC, McGillis SL, Greenspan JD (1996) Mechanical response properties of nociceptors innervating feline hairy skin. J Neurophysiol 75:1177-1189.

Gee MD, Lynn B, Cotsell B (1996) Activity-dependent slowing of conduction velocity provides a method for identifying different functional classes of C-fibre in the rat saphenous nerve. Neuroscience 73:667-675.

Gold MS, Reichling DB, Shuster MJ, Levine JD (1996) Hyperalgesic agents increase a tetrodotoxin-resistant $\mathrm{Na}^{+}$current in nociceptors. Proc Natl Acad Sci USA 93:1108-1112.

Grafe P, Quasthoff S, Grosskreutz J, Alzheimer C (1997) Function of the hyperpolarization-activated inward rectification in nonmyelinated peripheral rat and human axons. J Neurophysiol 77:421-426.

Hagbarth KE, Hallin RG, Hongell A, Torebjörk HE, Wallin BG (1972) General characteristics of sympathetic activity in human skin nerves. Acta Physiol Scand 84:164-176.

Hallin RG, Torebjörk HE (1974) Single unit sympathetic activity in human skin nerves during rest and various manoeuvres. Acta Physiol Scand 92:303-317.

Jansen JK, Nicholls JG (1973) Conductance changes, an electrogenic pump and the hyperpolarization of leech neurones following impulses. J Physiol (Lond) 229:635-655.

Koppert W, Zeck S, Sittl R, Likar R, Knoll R, Schmelz M (1998) Low-dose lidocaine suppresses experimentally induced hyperalgesia in humans. Anesthesiology 89:1345-1353.

Lynn B, Schutterle S, Pierau FK (1996) The vasodilator component of neurogenic inflammation is caused by a special subclass of heatsensitive nociceptors in the skin of the pig. J Physiol (Lond) 494:587-593.

Magerl W, Westerman RA, Mohner B, Handwerker HO (1990) Properties of transdermal histamine iontophoresis: differential effects of season, gender, and body region. J Invest Dermatol 94:347-352.

Meyer RA, Campbell JN (1981) Evidence for two distinct classes of unmyelinated nociceptive afferents in monkey. Brain Res 224:149-152.

Rang HP, Ritchie JM (1968) On the electrogenic sodium pump in mammalian non-myelinated nerve fibres and its activation by various external cations. J Physiol (Lond) 196:183-221.

Raymond SA, Lettvin JY (1978) Aftereffects of activity in peripheral axons as a clue to nervous coding. In: Physiology and pathobiology of axons (Waxman SG, ed), pp 203-225. New York: Raven.

Raymond SA, Thalhammer JG, Popitz BF, Strichartz GR (1990) Changes in axonal impulse conduction correlate with sensory modality in primary afferent fibers in the rat. Brain Res 526:318-321.

Sangameswaran L, Delgado SG, Fish LM, Koch BD, Jakeman LB, Stewart GR, Sze P, Hunter JC, Eglen RM, Herman RC (1996) Structure and function of a novel voltage-gated, tetrodotoxin-resistant sodium channel specific to sensory neurons. J Biol Chem 271:5953-5956.

Schaible HG, Schmidt RF (1988) Time course of mechanosensitivity changes in articular afferents during a developing experimental arthritis. J Neurophysiol 60:2180-2195.

Schmelz M, Schmidt R, Bickel A, Handwerker HO, Torebjörk HE (1997a) Specific C-receptors for itch in human skin. J Neurosci 17:8003-8008.

Schmelz M, Schmidt R, Bickel A, Handwerker HO, Torebjörk HE (1997b) Differential sensitivity of mechanosensitive and -insensitive C-fibers in human skin to tonic pressure and capsaicin. Soc Neurosci Abstr 23:1004.

Schmelz M, Schmidt R, Bickel A, Handwerker HO, Torebjörk HE (1999) Different activation patterns after capsaicin injection in subpopulations of $\mathrm{C}$-nociceptors in human skin. Brain, in press.

Schmidt R, Schmelz M, Forster C, Ringkamp M, Torebjörk HE, Handwerker HO (1995) Novel classes of responsive and unresponsive C nociceptors in human skin. J Neurosci 15:333-341.

Scholz A, Kuboyama N, Hempelmann G, Vogel W (1998) Complex 
blockade of TTX-resistant $\mathrm{Na}^{+}$currents by lidocaine and bupivacaine reduce firing frequency in DRG neurons. J Neurophysiol 79:1746-1754.

Serra J, Campero M, Ochoa J, Bostock H (1999) Activity-dependent slowing of conduction differentiates functional subtypes of $\mathrm{C}$ fibres innervating human skin. J Physiol (Lond) 515:799-811.

Thalhammer JG, Raymond SA, Popitz Bergez FA, Strichartz GR (1994) Modality-dependent modulation of conduction by impulse activity in functionally characterized single cutaneous afferents in the rat. Somatosens Mot Res 11:243-257.

Torebjörk HE (1974) Afferent C units responding to mechanical, thermal and chemical stimuli in human non-glabrous skin. Acta Physiol Scand 92:374-390.

Torebjörk HE, Hallin RG (1970) C-fibre units recorded from human sensory nerve fascicles in situ. A preliminary report. Acta Soc Med Ups 75:81-84.

Torebjörk HE, Hallin RG (1974) Responses in human A and C fibres to repeated electrical intradermal stimulation. J Neurol Neurosurg Psychiatry 37:653-664.

Trezise DJ, John VH, Xie XM (1998) Voltage- and use-dependent inhibition of $\mathrm{Na}+$ channels in rat sensory neurones by 4030W92, a new antihyperalgesic agent. Br J Pharmacol 124:953-963.

Van Essen D (1973) The contribution of membrane hyperpolarization to adaptation and conduction block in sensory neurones of the leech. J Physiol (Lond) 230:509-534.

Weidner C, Schmidt R, Hansson B, Schmelz M, Handwerker HO, Torebjörk HE (1998) Different axonal properties of mechanosensitive and mechanoinsensitive C-nociceptors. Soc Neurosci Abstr 24:383. 